\title{
The luminous infrared composite Seyfert 2 galaxy NGC 7679 through the [O III] $\lambda 5007$ emission line ${ }^{\star}$
}

\author{
I. M. Yankulova ${ }^{1}$, V. K. Golev ${ }^{1}$, and K. Jockers ${ }^{2}$ \\ 1 Department of Astronomy, St Kliment Okhridski University of Sofia, 5 James Bourchier Street, 1164 Sofia, Bulgaria \\ e-mail: [yan; valgol]@phys .uni-sofia.bg \\ 2 Max-Planck-Institut für Sonnensystemforschung, Max-Planck-Straße 2, 37191 Katlenburg-Lindau, Germany \\ e-mail: jockers@linmpi.mpg.de
}

Received 8 March 2007 / Accepted 29 March 2007

\begin{abstract}
Context. NGC 7679 (Mrk 534) is a nearby $(z=0.0177)$ nearly face-on SB0 luminous infrared Sy2 galaxy in which starburst and AGN activities co-exist. The ionization structure is maintained by both the AGN power-law continuum and starburst. The galaxy is a bright X-ray source possessing a low X-ray column density $N_{\mathrm{H}}<4 \times 10^{20} \mathrm{~cm}^{-2}$.

Aims. The Compton-thin nature of such unabsorbed objects infers that the simple formulation of the Unified model for SyGs is not applicable in their case. The absorption is likely to originate at larger scales instead of the pc-scale molecular torus. The main goal of this article is to investigate both gas distribution and ionization structure in the circumnuclear region of NGC 7679 in search for the presence of a hidden Sy1-type nucleus, using the [O III] $\lambda 5007$ luminosity as a tracer of AGN activity.

Methods. NGC 7679 was observed with the $2 \mathrm{~m}$ RCC reflector of the Ukraine National Astronomical Observatory at peak Terskol, Caucasus, Russia. The observations were carried out in October 1996 with the Focal Reducer of the Max-Planck-Institut für Sonnensystemforschung, Germany. All observations were taken with tunable Fabry-Perot narrow-band imaging with spectral $F W H M$ of the Airy profile $\delta \lambda$ between 3 and $4 \AA$ depending on the used wavelength.

Results. The [O III] $\lambda 5007$ emission-line image of the circumnuclear region of NGC 7679 shows elliptical isophotes extended along the $\mathrm{PA} \approx 80^{\circ}$ in the direction of the counterpart galaxy NGC 7682. There is a maximum of this emission which is shifted $\sim 4$ arcsec from the center as defined by the continuum emission. The maximum of ionization by the AGN power-law continuum traced by [O III] $] \lambda 5007 / \mathrm{H} \alpha$ ratio is displaced by $\sim 13$ arcsec eastward from the nucleus. The direction where high ionization is observed at $\mathrm{PA} \approx 80^{\circ} \pm 10^{\circ}$ coincides with the direction to the companion galaxy NGC $7682\left(\mathrm{PA} \approx 72^{\circ}\right)$. On the contrary, at PA $\sim 0^{\circ}$ the ionization in the circumnuclear region is entirely due to hot stars.

Conclusions. Both the ratio $\left(N_{\mathrm{ph}} / N_{\mathrm{ion}}\right)_{h v>55 \mathrm{eV}} \approx 0.2-20$ of the number $N_{\mathrm{ph}}$ of photons traced by [O III] to the number $N_{\text {ion }}$ of highenergy ionizing photons and the presence of weak and elusive $\mathrm{H} \alpha$ broad wings indicate a hidden AGN. We conclude that the dust and gas in the high ionization direction $\mathrm{PA} \approx 80^{\circ}$ has a direct view to the central AGN engine. This possibly results in dust/star-formation decay. A large fraction of the unabsorbed Compton-thin Sy2s with [O III] luminosity $\gtrsim 10^{41} \mathrm{erg} \mathrm{s}^{-1}$ possesses a hidden AGN source.
\end{abstract}

Key words. galaxies: individual: NGC 7679 (Mrk 534) - galaxies: ISM - galaxies: starburst - galaxies: Seyfert

\section{Introduction}

Luminous infrared galaxies (LIGs) are characterized by extreme IR luminosities $L_{\mathrm{IR}} \gtrsim 10^{11} L_{\odot}$ at mid- to far-infrared (FIR) wavelengths. In their comprehensive spectroscopic survey of LIGs Kim et al. (1995) and Veilleux et al. (1995) have shown a clear tendency for the more luminous objects to be more Seyfert-like. The starburst and AGN are tightly connected phenomena and the interaction between them is a matter of debate.

Based on a large spectroscopic optical survey of bright IRAS and X-ray sources from ROSAT All Sky Survey, Moran et al. (1996) extracted low-redshift galaxies with optical spectra characterized by the HII regions and X-ray luminosities typical of AGNs and these objects were named Composite Seyfert/Starburst galaxies. Other similar galaxies (i.e. with bright X-ray emission together with the clear predominance of a starburst in the optical and IR regime) have been found also in the deep ROSAT fields (Boyle et al. 1995; Griffiths et al. 1996)

^ Based on observations obtained at the Peak Terskol Observatory, Caucasus, Russia. and in the Chandra and XMM-Newton deep fields (Rosati et al. 2001).

A significant part of the observed FIR-emission of these composites could be associated with circumnuclear starburst events. The nuclear X-ray source there is generally absorbed with column density of $N_{\mathrm{H}}>10^{22} \mathrm{~cm}^{-2}$ and these values range from $10^{22} \mathrm{~cm}^{-2}$ to higher than $10^{24} \mathrm{~cm}^{-2}$ for about $96 \%$ of this class of objects (Risaliti et al. 1999; Bassani et al. 1999). The circumnuclear starburst should also play a major role in the obscuration processes - see for details Levenson et al. (2001) and references therein. However, there are Sy 2 galaxies with column densities lower than $10^{22} \mathrm{~cm}^{-2}$. Panessa \& Bassani (2002, hereafter PB02) present a sample of 17 type-2 SyGs showing such low absorption in X-rays. The Compton thin nature of these sources is strongly suggested by some isotropic indicators such as FIR and [O III] emission.

The fraction of Composite Seyfert/Starburst objects is estimated to be in the range of $10 \%-30 \%$ of the Sy 2 population. The simple formulation of the Unified model for SyGs is not applicable in such sources. The observed absorption is likely to originate instead at larger scales in the pc-scale molecular torus. 
Probably the Broad Line Regions (BLRs) of these objects are covered by some obscuring dusty material.

NGC 7679 is a nearby $(z=0.0177)$ nearly face-on SB0 Seyfert 2 type galaxy in which starburst and AGN activities coexist. The IRAS fluxes show that the luminosity of NGC 7679 in the far infrared is about $L_{\mathrm{FIR}} \approx 10^{11} L_{\odot}$. This object is included in the large spectroscopic survey of 200 luminous IRAS galaxies (Kim et al. 1995; Veilleux et al. 1995). NGC 7679 is physically associated by a common stream of ionized gas with the Sy2 galaxy NGC 7682 at $\sim 4.5$ arcmin eastward $\left(\mathrm{PA} \approx 72^{\circ}\right.$ ) forming the pair Arp 216 (VV 329). The tidal interactions between both galaxies together with the existence of a bar in NGC 7679 could enhance the gas flow towards the nuclear regions and possibly trigger the starburst processes $(\mathrm{Gu}$ et al. 2001).

The X-ray properties of the NGC 7679 based on the BeppoSAX observations and on the ASCA archive were discussed by Della Ceca et al. (2001, hereafter DC01). Their conclusion is that NGC 7679 is a Seyfert-starburst composite galaxy which implies the clear predominance of an AGN in the X-ray regime connected with a starburst in the optical and IR regime. DC01 found that a simple power-law spectral model with $\Gamma \sim$ 1.75 and small intrinsic absorption $\left(N_{\mathrm{H}}<4 \times 10^{20} \mathrm{~cm}^{-2}\right)$ provides a good description of the spectral properties of NGC 7679 from 0.1 to $50 \mathrm{keV}$. The small X-ray absorption and the absence of strong $(E W \sim 1 \mathrm{keV}) \mathrm{Fe}$-lines suggest a Compton thin type-2 AGN in NGC 7679, which clearly distinguishes this galaxy from the other LIG Seyferts.

The main goal of this article is to investigate both gas distribution and ionization structure in the circumnuclear regions of the luminous IR unabsorbed Seyfert galaxy NGC 7679 and to look for tracers of the presence of a hidden Sy1-type nucleus.

Some information on the observations and data reduction procedures is presented in Sect. 2. The results are presented in Sect. 3 and discussed in Sect. 4. The combination of the data taken from recent literature and our Fabry-Perot observations provides new insight in the circumnuclear region of NGC 7679 and in the phenomena occurring there.

\section{Observations and data reduction}

NGC 7679 was observed by K. Jockers, T. Bonev, and T. Credner with the $2 \mathrm{~m} \mathrm{RCC}$ reflector of the Peak Terskol Observatory, Caucasus, Russia. The observations were carried out in October 1996 with the Two-channel Focal Reducer of the former Max-Planck-Institut für Aeronomie, Germany (now Max-Planck-Institut für Sonnensystemforschung, MPS). This instrument was primarily intended for cometary studies, but it has repeatedly been used for observations of active galactic nuclei (see for example Golev et al. 1995, 1996; and Yankulova 1999). The technical data and the present capabilities of the MPS Two-channel Focal Reducer are described in Jockers (1997) and Jockers et al. (2000).

All observations were taken in Fabry-Perot (FP) mode using tunable FP narrow-band imaging with spectral FWHM of the Airy profile $\delta \lambda$ in order of 3-4 $\AA$. The details of observations are presented in Table 1 where the central wavelengths $\lambda_{\mathrm{c}}$ and the effective width $\Delta \lambda$ of the interference filters used to separate the Fabry-Perot interference orders, the wavelength $\lambda_{\mathrm{FP}}$ at which the Fabry-Perot was tuned, and the exposures are listed.

The overall "finesse" of the system $\Delta \lambda / \delta \lambda$ is $\approx 15, \Delta \lambda$ is the free spectral range of the FP. As one can see from Table 1, $\Delta \lambda$ is comparable to the filter's band width and therefore all FP orders except the central one are efficiently suppressed. Two
Table 1. Observiation details.

\begin{tabular}{llll}
\hline \hline $\begin{array}{l}\text { image } \\
\text { frame }\end{array}$ & $\begin{array}{l}\text { interference }_{\text {filter }^{a}} \\
\lambda_{\mathrm{c}} / F W H M \\
(\AA) /(\AA)\end{array}$ & $\begin{array}{l}\text { Fabry-Perot } \\
\text { tuned } \\
\text { wavelength } \\
\lambda_{\mathrm{FP}} \\
(\AA)\end{array}$ & $\begin{array}{l}\text { frames } \\
\times \\
\text { exposure } \\
\text { time } \\
(\mathrm{s})\end{array}$ \\
\hline $\mathrm{H} \alpha$ & $6662 / 55$ & 6674.8 & $1 \times 1800$ \\
& & & $2 \times 900$ \\
{$[\mathrm{~N} \mathrm{II}] \lambda 6548$} & $6662 / 55$ & 6659.9 & $1 \times 900$ \\
continuum & $6719 / 33$ & 6720.0 & $1 \times 1800$ \\
& & & $1 \times 900$ \\
{$[\mathrm{O} \mathrm{III}] \lambda 5007$} & $5094 / 44$ & 5092.4 & $2 \times 900$ \\
continuum & $5002 / 41$ & 4437.7 & $1 \times 1200$ \\
Gunn $\mathrm{r}^{b}$ & $6800 / 1110$ & & $2 \times 60$ \\
$\mathrm{BG} \mathrm{39/2}{ }^{b}$ & $4720 / 700$ & & $2 \times 1500$ \\
\hline
\end{tabular}

a Used to separate Fabry-Perot working orders.

$b$ Broad-band image taken without Fabry-Perot to reveal the morphology.

exposures of NGC 7679 were obtained through each filter to eliminate cosmic ray events and to increase the signal-to-noise ratio. Flat field exposures were obtained using dusk and dawn twilight for uniform illumination of the detector. No dark correction was required.

The images were reduced following the usual reduction steps for narrow-band imaging. After flatnfielding, the frames were aligned by rebinning to a common origin. The final alignment of all the images was estimated to be better than $0.1 \mathrm{px}$ (the scale is $1 \mathrm{px}=0.8$ arcsec). A convolution procedure was performed in order to match the Point-Spread Functions (PSFs) of each linecontinuum pair which unavoidably degrades the final FWHM of the images to the mean value $\approx 3-3.3$ arcsec (shown as "seeing" in Fig. 1). At the distance of NGC 7679, one arcsec corresponds to a distance of about $340 \mathrm{pc}$ assuming $H_{0}=75 \mathrm{~km} \mathrm{~s}^{-1} \mathrm{Mpc}^{-1}$.

\section{Results}

\subsection{Narrow-band emission-line images}

Gray-scale images of the narrow-band flux distribution of the extended circumnuclear region of NGC 7679 in the [O III $] \lambda 5007$, $\mathrm{H} \alpha$, and $[\mathrm{N} \mathrm{II}] \lambda 6548$ emission lines with superimposed contours are presented in Fig. 1-3, respectively.

The [O III] $\lambda 5007$ emission shown in Fig. 1 reveals a bright, about 20 arcsec in size, extended emission-line region (EELR) which is elongated approximately in East direction $\left(\mathrm{PA} \approx 80^{\circ} \pm\right.$ $10^{\circ}$ ). This region is similar to the analogous EELRs observed in many Sy2 type galaxies. Most probably it is powered by the AGN-type activity of the nucleus. The emission-line peak of [O III] $\lambda 5007$ is shifted at about $\sim 4$ arcsec to the East with respect to the center defined by the continuum emission and marked by cross in Fig. 1.

At larger distances $(\sim 37 \mathrm{arcsec})$ the ionized gas forms an envelope which is extended along the direction $\mathrm{PA} \approx 72^{\circ}$ to the NGC 7682, the counterpart of NGC 7679, as was already noted by Durret \& Warin (1990).

In Fig. 2 we present our very deep and high-contrast $\mathrm{H} \alpha$ continuum-subtracted image with numerous starburst regions where, because of both seeing and pixel size, we are able to see only elliptical central isophotes instead of the "double nucleus" observed recently by Buson et al (2006). Our analysis of the unpublished $\mathrm{H} \alpha$ images taken from the archive of 


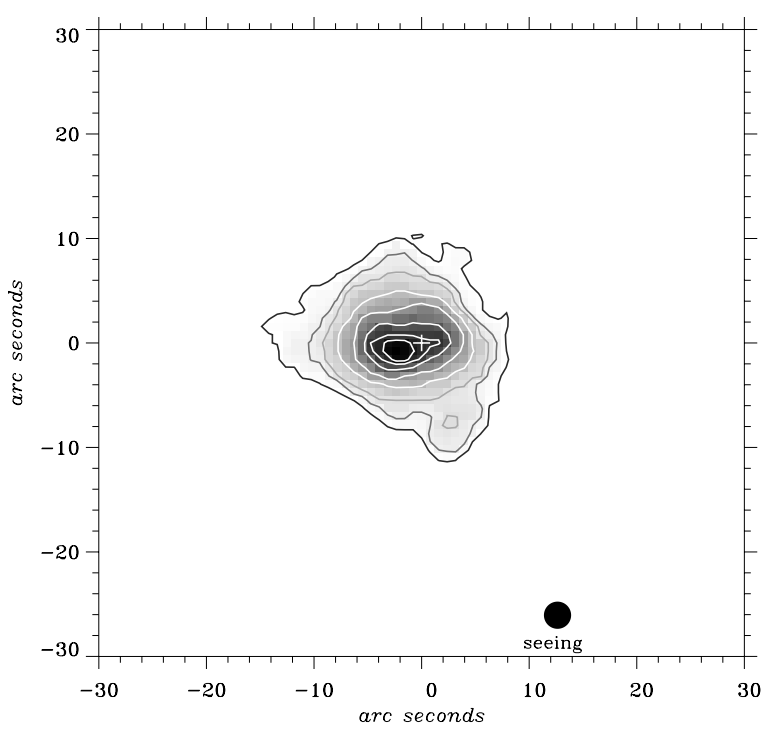

Fig. 1. Contours of continuum-subtracted narrow-band [O III] $] \lambda 5007$ image superimposed on the gray-scale [O III] $\lambda 5007$-emission distribution of the circumnuclear region of NGC 7679. The background noise level is $\sigma=2.01 \times 10^{-17} \mathrm{erg} \mathrm{cm}^{-2} \mathrm{~s}^{-1} \operatorname{arcsec}^{-2}$. The outermost contour is taken at $5 \sigma$ above the sky level and the next contours increase by a factor of $\sqrt{2}$. Note East-West elongation and two extrema decentered of about $\sim 4$ arcsec from the position of the nucleus marked by cross. North is up, East is to the left.

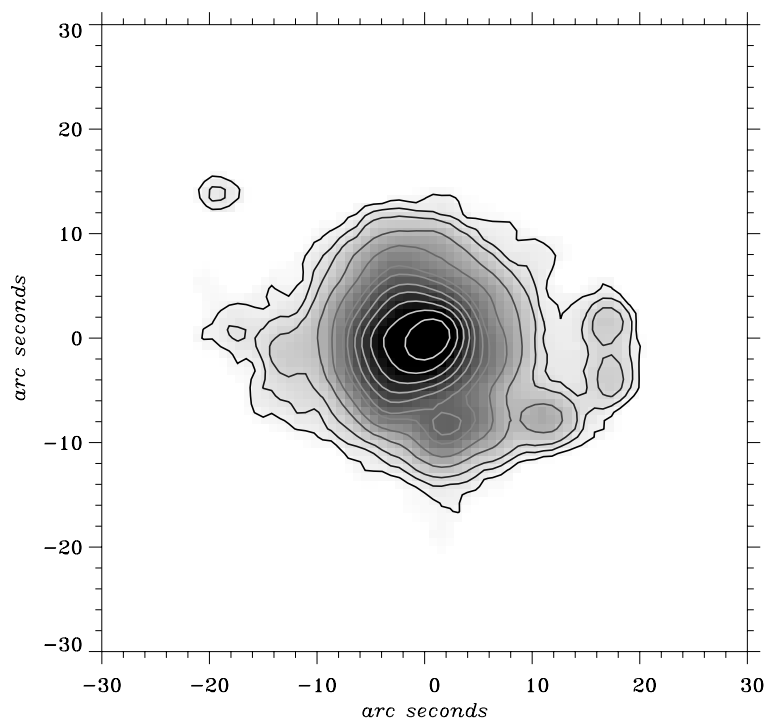

Fig. 2. Contours of continuum-subtracted narrow-band $\mathrm{H} \alpha$-image superimposed on the gray-scale $\mathrm{H} \alpha$-emission distribution of the circumnuclear region of NGC 7679. The background noise level is $\sigma=$ $2.77 \times 10^{-18} \mathrm{erg} \mathrm{cm}^{-2} \mathrm{~s}^{-1} \operatorname{arcsec}^{-2}$. The outermost contour is taken at $5 \sigma$ above the sky level and the next contours increase by a factor of $\sqrt{2}$. North is up, East is to the left.

the Isaak Newton Group of telescopes at La Palma as well as the archive images of Buson et al. (2006) from the ESO La Silla NTT also revealed a "double nucleus" otherwise unseen in the known broad-band images. The separation between the nuclear counterparts (in fact one is the active nucleus itself and the other one is a bright, spiral-like, extremely powerful starburst region) is $\approx 3$ arcsec. The existence of this "double nucleus" in NGC 7679 could enhance the gas flows towards the nuclear regions and possibly trigger the starburst process itself.

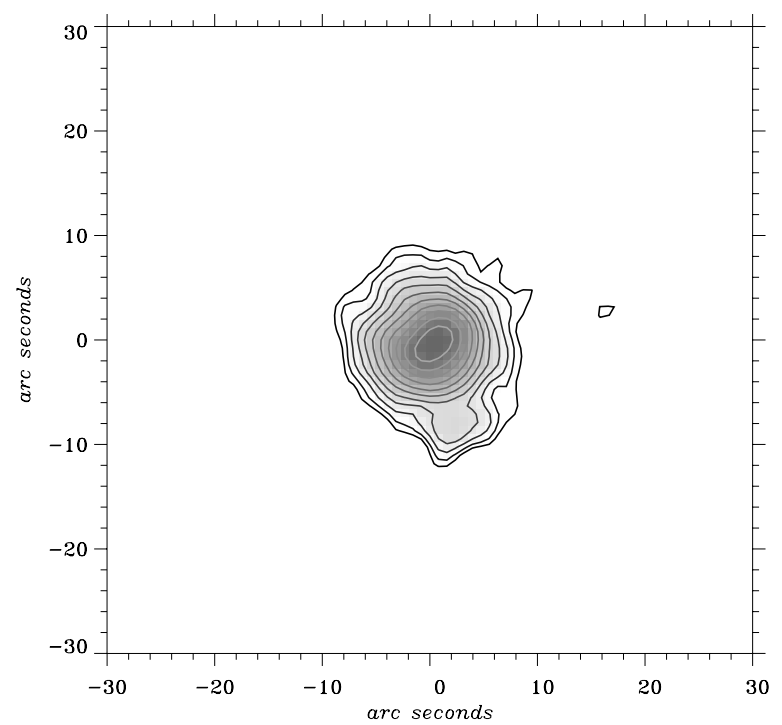

Fig. 3. Contours of continuum-subtracted narrow-band [N II] $\lambda 6548$ image superimposed on the gray-scale [N II] $\lambda 6548$-emission distribution of the circumnuclear region of NGC 7679. The background noise level is $\sigma=4.75 \times 10^{-18} \mathrm{erg} \mathrm{cm}^{-2} \mathrm{~s}^{-1} \operatorname{arcsec}^{-2}$. The outermost contour is taken at $5 \sigma$ above the sky level and the next contours increase by a factor of $\sqrt{2}$. North is up, East is to the left.

The "double nucleus" can be also seen at a very different wavelength range on $6 \mathrm{~cm}$ and $20 \mathrm{~cm}$ high-resolution VLA radio continuum map of NGC 7679 published by Stine (1992). The angular distance and PA between two counterparts is quite the same. The radio spectral index is -0.37 and steepens away from the center which indicates that nonthermal emission leaks out of the starburst region.

The low-excitation gas traced by the emission in $\mathrm{H} \alpha$ reveals different morphology as compared to that of the [O III] $\lambda 5007$ emission. Inside of the region with a radius of 6-8 arcsec from the center, the contours of the $\mathrm{H} \alpha$ emission are nearly circular. Outside this region to the West of the main body of NGC 7679 a clearly outlined wide arc is observed at 16 arc$\sec (\sim 5 \mathrm{kpc})$ from the center. To the East this arc converts into a gaseous envelope which forms a part of a circumnuclear starforming ring mentioned by Pogge (1989). This arc is not detected on the narrow-band continuum image next to the $\mathrm{H} \alpha$. The same morphology in $\mathrm{H} \alpha+[\mathrm{N} \mathrm{II}]$ with higher spatial resolution was observed by Buson et al. (2006).

The Fabry-Perot technique we use makes possible to disentangle [N II] $\lambda 6548$ from $\mathrm{H} \alpha$. The pure [N II] $\lambda 6548$ emission (Fig. 3) shows extended structure $\sim 20$ arcsec in diameter. The starforming ring revealed by the $\mathrm{H} \alpha$ image is not seen here. As a rule, the gas component in the starforming ring is ionized by stellar UV-emission and the [N II] $\lambda 6548$ is weaker than one where the gas is ionized by power-low AGN continuum. On the other hand, this could be an effect due to the shorter exposure time of our [N II] $\lambda 6548$ frame.

\subsection{Narrow-band emission-line total fluxes}

The total emission-line fluxes of $\mathrm{H} \alpha$, [O III] $\lambda 5007$ and [N II] $\lambda 6583$ were estimated from our flux-calibrated images in an aperture of $2 \mathrm{kpc}(r \lesssim 3 \mathrm{arcsec})$ like the one used by the authors cited in Table 2 . In this table we collected available measurements of the emission lines we observed. Our measured fluxes are in good agreement with those of Kim et al. (1995) and 
Table 2. Measured emission lines fluxes in $2 \mathrm{kpc}$ central aperture in NGC 7679.

\begin{tabular}{|c|c|c|c|c|c|c|c|}
\hline \multirow[t]{3}{*}{ Emission } & \multicolumn{7}{|c|}{ Measured flux $F(\lambda), \mathrm{erg} \mathrm{cm}^{-2} \mathrm{~s}^{-1}$} \\
\hline & \multicolumn{5}{|c|}{$2 \mathrm{kpc}$ central aperture } & \multicolumn{2}{|c|}{9 arcsec off the nucleus } \\
\hline & 1 & 2 & 3 & 4 & 5 & 6 & 7 \\
\hline $\mathrm{H} \alpha$ & $1.92 \times 10^{-13}$ & $1.9 \times 10^{-13}$ & $4.5 \times 10^{-13}$ & - & $3.8 \times 10^{-13}$ & $3.73 \times 10^{-14}$ & $1.04 \times 10^{-14}$ \\
\hline$[\mathrm{N}$ II] $] \lambda 6548$ & $9.96 \times 10^{-14}$ & $1.08 \times 10^{-13}$ & $1.86 \times 10^{-13}$ & - & - & $9.8 \times 10^{-15}$ & $4.5 \times 10^{-15}$ \\
\hline$[\mathrm{O}$ III $] \lambda 5007$ & $5.2 \times 10^{-14}$ & $5.3 \times 10^{-14}$ & $8.8 \times 10^{-14}$ & - & - & $9 \times 10^{-15}$ & $4.6 \times 10^{-15}$ \\
\hline $\mathrm{H} \beta$ & - & $1.1 \times 10^{-14}$ & $5.24 \times 10^{-14}$ & $1.0 \times 10^{-14}$ & - & $5.9 \times 10^{-15}$ & - \\
\hline$F(\mathrm{H} \alpha) / F(\mathrm{H} \beta)$ & - & 17.4 & 8.5 & 5.0 & 4.58 & 6.3 & - \\
\hline$F(\mathrm{H} \gamma) / F(\mathrm{H} \beta)$ & - & 0.24 & 0.32 & 0.4 & 0.3 & - & - \\
\hline$C$ & - & 4.93 & 2.88 & 1.6 & 1.12 & 2.02 & - \\
\hline$E(B-V)$ & - & 1.45 & 0.85 & 0.47 & 0.33 & 0.65 & - \\
\hline
\end{tabular}

Columns: 1 - this work; 2 - Kim et al. (1995); 3 - Contini et al. (1998); 4 - Kewley et al. (2000); 5 - Buson et al. (2006); 6 - Contini et al. (1998); 7 - this work, $\mathrm{PA}=207^{\circ}$.

differ from the measurements of Contini et al. (1998). Flux values given by Contini et al. (1998) are twice as large as ours and those given by Kim et al. (1995).

Recently $\mathrm{Gu}$ et al. (2006) measured the central flux in [O III] $\lambda 5007$. We found a reasonable coincidence between their value $\left(1.55 \times 10^{-14} \mathrm{erg} \mathrm{cm}^{-2} \mathrm{~s}^{-1}\right)$ and ours $(1.94 \times$ $\left.10^{-14} \mathrm{erg} \mathrm{cm}^{-2} \mathrm{~s}^{-1}\right)$ in the much smaller aperture they used.

We estimated the flux of the continuum near [O III] $\lambda 5007$ within the central $2 \mathrm{kpc}$ to be $F\left(\lambda_{\text {cont }}\right)=6.74 \times$ $10^{-15} \mathrm{erg} \mathrm{cm}^{-2} \mathrm{~s}^{-1} \AA$. Then the equivalent width of the emission line [O III] $\lambda 5007$ is $E W(\lambda 5007)=7.6 \AA$. Baskin \& Loar (2005) used the photoionization code CLOUDY to calculate the dependence of $E W(\lambda 5007)$ on the electron density $n_{\mathrm{e}}$, the ionization parameter $U$, and the covering factor $C F$. Following their Fig. 5 and our estimation of $E W(\lambda 5007)$ we derive for the covering factor $C F$ the range $0.016 \leq C F \leq 0.04$ with the most probable value $C F \approx 0.024$.

There is a large quantity of absorbing matter in the central region of NGC 7679 (Telesco et al. 1995) which modifies the Balmer emission lines. The Balmer decrement reported by Kim et al. (1995) in the central $2 \mathrm{kpc}$ is $F(\mathrm{H} \alpha) / F(\mathrm{H} \beta) \approx 17.4$, but following Contini et al. (1998) this decrement is 8.5. Kewley et al. (2000) give $E(B-V)=0.47$ which results in $F(\mathrm{H} \alpha) / F(\mathrm{H} \beta)=$ 5.04. In Table 2 the value of the parameter $C$ is evaluated from the measured Balmer decrement and from the assumption that in AGNs $F(\mathrm{H} \alpha) / F(\mathrm{H} \beta)=3.1$ and the optical depth $\tau_{\lambda}=C f(\lambda)$ where $f(\lambda)$ is the reddening curve (Osterbrock 1989). The extinction $E(B-V)$ derived from the Balmer decrement is also given in Table 2.

Contini et al. (1998) present measurements of emission-lines fluxes made in the extranuclear region 9 arcsec off the nucleus at $\mathrm{PA}=207^{\circ}$ in an aperture of 3 arcsec. We estimated the emissionline fluxes from our images in the same aperture at the same place in order to compare with those given by Contini et al. (1998). The results are given in Table 2. Contini's values are about 2 times larger than ours in the extranuclear region as well as at the nucleus.

Moustakas \& Kennicutt (2006) report total emission-line fluxes of $\mathrm{H} \alpha$ and $[\mathrm{O} \mathrm{III}] \lambda 5007$ in a wide rectangular aperture $30 \times 80$ arcsec oriented at $\mathrm{PA}=90^{\circ}$. Their $\mathrm{H} \alpha$-flux $F(\mathrm{H} \alpha)=$ $(1.535 \pm 0.062) \times 10^{-12} \mathrm{erg} \mathrm{cm}^{-2} \mathrm{~s}^{-1}$ coincides with our value $\left(1.52 \times 10^{-12} \mathrm{erg} \mathrm{cm}^{-2} \mathrm{~s}^{-1}\right)$ in the same wide aperture after a correction for extinction with $E(B-V)=0.065$ used by them. In $[\mathrm{O}$ III $] \lambda 5007$ the coincidence is reasonably good $\left(4.72 \times 10^{-13}\right.$ compared with ours $3.90 \times 10^{-13} \mathrm{erg} \mathrm{cm}^{-2} \mathrm{~s}^{-1}$ ).

\subsection{The ionization map $F([\mathrm{O} I 11] \lambda / 5007 / F(H \alpha)$}

Our flux-calibrated emission-line images are used to form the $F([\mathrm{O}$ III] $\lambda 5007) / F(\mathrm{H} \alpha)$ ionization map in order to analyse the mean level of ionization. This map is shown in the left panel of Fig. 4. All pixels below $4 \sigma$ of the background noise level were suppressed before the division of the corresponding images. The ionization map infers a presence of a maximum shift to the East at $\mathrm{PA} \approx 80^{\circ}$ with respect to the photometric center defined by the integral light of the continuum images and marked by cross on the figure.

A slice of this map along the $\mathrm{PA} \approx 80^{\circ}$ versus the axial distance from the nucleus is presented in the right panel of Fig. 4. Below we will discuss in more detail the behaviour of the ionization at positions 1 to 5 .

\section{Discussion}

\subsection{The ionizing flux from the central engine}

In order to estimate the number of ionizing photons emitted from the central engine, we made use of the recent X-ray observations of NGC 7679. This object was observed by ASCA and BeppoSAX in 1998, and by XMM-Newton in 2005. A detailed analysis of ASCA and BeppoSAX data sets is present in DC01. They show that a single absorbed power-law function (with a photon index 1.75) fits the observed spectrum very well and the $\mathrm{X}$-ray absorption is relatively small $\left(N_{\mathrm{H}} \leq 4 \times 10^{20} \mathrm{~cm}^{-2}\right)$.

The data for the X-ray observations in 2005 were taken from the XMM-Newton public archive. The corresponding X-ray spectra for the PN and the two MOS detectors were extracted following the standard procedures using the XMM-Newton Science Analysis System software (SAS version 7.0.0). A single absorbed power-law function gave a good fit $\left(\chi^{2} /\right.$ d.o.f. $\left.=201 / 191\right)$ to all the three spectra which were fitted simultaneously. The small X-ray absorption in the nucleus of NGC 7679 was confirmed, $N_{\mathrm{H}}=5.6[4.0 \div 7.5] \times 10^{20} \mathrm{~cm}^{-2}$, and no change in the shape of the spectrum was found, a photon index of $1.81[1.70 \div$ 1.92] (the $90 \%$-confidence intervals are given in brackets). The absorbing X-ray column density along the line of sight is about an order of magnitude smaller than the one estimated from the observed Balmer decrement which is $N_{\mathrm{H}} \sim 8 \times 10^{21} \mathrm{~cm}^{-2}$ and $\sim 5 \times 10^{21} \mathrm{~cm}^{-2}$ following Kim et al. (1995) and Contini et al. (1998), respectively.

Interestingly, the observed X-ray flux decreased by a factor $\sim 10$ over a time period of $\sim 7$ years: $F_{X}=3.8 \times 10^{-13}$ and $5.8 \times$ $10^{-13} \mathrm{erg} \mathrm{cm}^{-2} \mathrm{~s}^{-1}$ correspondingly in the $0.1-2.0 \mathrm{keV}$ 

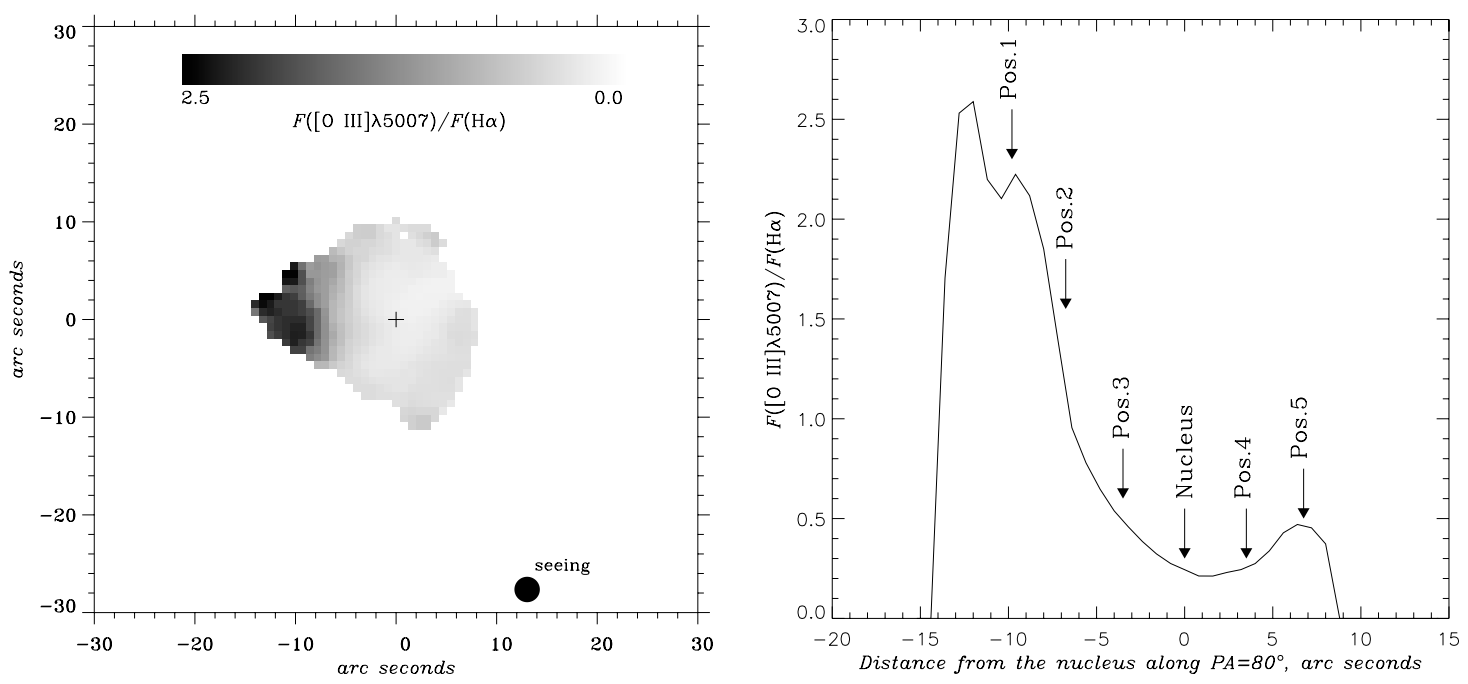

Fig. 4. $F([\mathrm{O} \mathrm{III}] \lambda 5007) / F(\mathrm{H} \alpha)$ ionization map of NGC 7679. All pixels below $4 \sigma$ of the background noise level were suppressed before image division (left). The ratio $F([\mathrm{O} \mathrm{III}] \lambda 5007) / F(\mathrm{H} \alpha)$ vs. the axial distance from the nucleus along $\mathrm{PA} \approx 80^{\circ}$ (right). The positions labeled 1 to 5 are equidistant with step size of 3 arcsec. We refer to them later in the text (see Fig. 6).

and $2.0-10.0 \mathrm{keV}$ energy intervals. Since, on the one hand, there is only about $5 \%$ scatter of the fluxes for all three detectors (one $\mathrm{PN}$ and two MOS) around the average values given above, and, on the other hand, NGC 7679 shows an appreciable X-ray variability (DC01), it is then likely that the detected decrease of the $\mathrm{X}$-ray flux is real and not an instrumental effect.

The extrapolation of the DC01's power law to the UV spectral domain (that is to $h v_{0}=13.6 \mathrm{eV}$ ) yields $F_{v}^{\mathrm{nt}}=F_{v_{0}}\left(v_{0} / v\right)^{\alpha}$ where $\alpha=0.75$ and $F_{\nu_{0}}=2.0 \times 10^{-28} \mathrm{erg} \mathrm{cm}^{-2} \mathrm{~s}^{-1} \mathrm{~Hz}^{-1}$. The same extrapolation for the XMM-Newton spectrum results in $F_{v}^{\mathrm{nt}}=F_{v_{0}}\left(v_{0} / v\right)^{\alpha}$ where $\alpha=0.81$ and $F_{v_{0}}=2.8 \times$ $10^{-29} \mathrm{erg} \mathrm{cm}^{-2} \mathrm{~s}^{-1} \mathrm{~Hz}^{-1}$.

The number of ionizing photons with $h v>55 \mathrm{eV}$ provided by the central AGN source is defined as

$N_{\text {ion }}=\int_{55 \mathrm{eV}}^{\infty} \frac{F_{v}^{\mathrm{nt}}}{h v} \mathrm{~d} v=4 \pi R_{G}^{2} \frac{F_{h v=55 \mathrm{eV}}^{\mathrm{nt}}}{h \alpha}$

where $R_{G}$ is the distance to the NGC 7679. For the BeppoSAX data this estimation is $N_{\text {ion }} \sim 10^{52} \mathrm{ph} \mathrm{s}^{-1}$ and for the XMMNewton data $N_{\text {ion }} \sim 10^{51} \mathrm{ph} \mathrm{s}^{-1}$. These values are averaged between all BeppoSAX and XMM-Newton bands, respectively. The number of ionizing photons decrease from the BeppoSAX time to the XMM-Newton time in the range of $10^{51} \lesssim N_{\text {ion }} \lesssim$ $10^{52} \mathrm{ph} \mathrm{s}^{-1}$.

\subsection{Physical conditions in the circumnuclear region of NGC 7679}

The extended emission-line region in NGC 7679 has a rather different morphology when observed in $\mathrm{H} \alpha$ (low ionization emission line) as compared to [O III] $\lambda 5007$ (high ionization emission line). The $\mathrm{H} \alpha$ image (Fig. 2) contains a compact circumnuclear region ( $\sim 20$ arcsec in diameter) whose isophotes do not infer any preferred direction. In contrast, the [O III $] \lambda 5007$ image (Fig. 1) of the circumnuclear region of NGC 7679 shows elliptical isophotes extended along the $\mathrm{PA} \approx 80^{\circ} \pm 10^{\circ}$. Such a difference in morphology of the emission-line images signals the presence of at least two distinct ionization components (see for example Pogge 1989).
The extended morphology both of the [O III $] \lambda 5007$ image (Fig. 1) and of the [O III] $\lambda 5007 / \mathrm{H} \alpha$ flux ratio image (Fig. 4) suggests an anisotropy of the radiation field. In order to check whether the ionizing field is collimated or not we have to compare the number of ionizing photons $N_{\mathrm{ph}}$, absorbed by the extended emission line gas, with the number of ionizing photons $N_{\text {ion }}$, emitted by the central AGN engine. Usually, the hydrogen line flux $F(\mathrm{H} \alpha)$ or $F(\mathrm{H} \beta$,$) is used to find N_{\mathrm{ph}}$. But the NGC 7679 high-resolution $\mathrm{H} \alpha$ image reveals a central circumnuclear star-forming spiral ring capable of producing about $\sim 75 \%$ of the optical line emission within a radius of $\sim 1 \mathrm{kpc}$ (Buson et al. 2006). For this reason it is not quite correct to use the $F(\mathrm{H} \alpha)$ in order to make the $N_{\mathrm{ph}}$ estimate.

Kauffmann et al. (2003) focus on the luminosity of the [O III] $\lambda 5007$ as a tracer of AGN activity. We can estimate the number $N_{\text {ph }}$ of ionizing photons with energy above $h v=$ $55 \mathrm{eV}$ from the observed [O III] $\lambda 5007$ luminosity after correction for extinction. A dust correction to [O III] based on the ratio $F(\mathrm{H} \alpha) / F(\mathrm{H} \beta)$ should be regarded as best approximation (Kauffmann et al. 2003). According to Draine \& Lee (1984) (Fig. 7 therein) the optical depth is $\tau_{5007}=0.96 C=2.76$. Here we adopt the value of $C=2.88$, following Contini et al. (1998), as a more compromising reddening value among the different Balmer decrement assessments. Then the luminosity, corrected for extinction, is $L^{\text {corr }}\left(\left[\mathrm{O}^{+2}\right] \lambda 5007\right)=4.4 \times 10^{41} \mathrm{erg} \mathrm{s}^{-1}$. We note that PB02 give $5.7 \times 10^{41} \mathrm{erg} \mathrm{s}^{-1}$ for the [O III] $\lambda 5007$ luminosity.

The total number of ionizing photons that must be available to produce the observed [O III] $\lambda 5007$ emission is given by the expression

$$
\begin{aligned}
N_{\mathrm{ph}} & =\frac{\alpha_{G}\left(\mathrm{O}^{+2}, T_{\mathrm{e}}\right) L^{\mathrm{corr}}\left(\left[\mathrm{O}^{+2}\right] \lambda 5007\right) C F^{-1}}{\alpha_{5007}^{\mathrm{eff}}\left(n_{\mathrm{e}}, T_{\mathrm{e}}\right) h v_{5007}} \\
& \approx 2 \times 10^{52} \mathrm{ph} \mathrm{s}^{-1}
\end{aligned}
$$

where $\alpha_{G}\left(\mathrm{O}^{+2}, T_{\mathrm{e}}\right)=5.1 \times 10^{-12} \mathrm{~cm}^{3} \mathrm{~s}^{-1}$ (Aldrovandi \& Pequignot 1973) is the recombination coefficient at $T_{\mathrm{e}} \approx 10^{4} \mathrm{~K}$ and $\alpha_{5007}^{\text {eff }}\left(n_{\mathrm{e}}, T_{\mathrm{e}}\right)=1.1 \times 10^{-9} \mathrm{~cm}^{3} \mathrm{~s}^{-1}$ is the effective recombination coefficient at $n_{\mathrm{e}}=10^{5} \mathrm{~cm}^{-3}$ and $T_{\mathrm{e}}=10^{4}$ $\mathrm{K}$. This coefficient strongly depends on the electron density and temperature. If we accept $T_{\mathrm{e}}=10^{4} \mathrm{~K}$ then $\alpha_{5007}^{\text {eff }}\left(n_{\mathrm{e}}\right)=$ $5.14 \times 10^{-3} A_{21} / n_{\mathrm{e}} \mathrm{cm}^{3} \mathrm{~s}^{-1}$ where $A_{21}=0.021 \mathrm{~s}^{-1}$. As the critical 


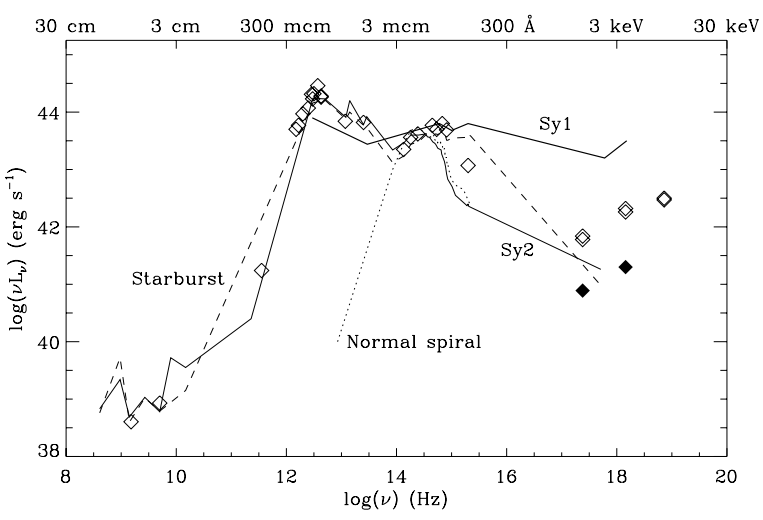

Fig. 5. Spectral energy distribution (SED) from the radio to the X-ray band of the composite Starburst/Sy2 galaxy NGC 7679 (open diamonds). The radio values at $6 \mathrm{~cm}$ and $20 \mathrm{~cm}$ are from VLA (Stine 1992). The X-ray band data are from ASCA and BeppoSAX (DC02 and Risaliti 2002). Filled diamonds represent recent X-ray observations taken from the XMM-Newton archive. All other data are taken from NED. The SED has been compared with a normal spiral galaxy template (dotted line) taken from Elvis et al. (1994), with Starburst and Sy2 galaxy templates (dashed line and thin solid line) taken from Schmitt et al. (1997), and with Sy1 galaxy template (thick solid line) taken from Mas-Hesse et al. (1995).

electron density is $n_{\mathrm{e}}^{\mathrm{cr}}(5007)=5 \times 10^{5} \mathrm{~cm}^{-3}$ we assume that the electron density is not lower than $n_{\mathrm{e}} \approx 10^{4} \mathrm{~cm}^{-3}$ in order to emit the [O III] 25007 . Then the lower limit for $N_{\mathrm{ph}}$ is $\approx 2 \times 10^{51} \mathrm{ph} \mathrm{s}^{-1}$. For NGC 7679 the covering factor $\mathrm{CF}=0.024$.

The photon ratio $N_{\mathrm{ph}} / N_{\text {ion }}$ is a probe of the collimation hypothesis. In the anisotropic case this ratio is considerably larger than 1 . Under the above assumptions about $n_{\mathrm{e}}$ and $T_{\mathrm{e}}$ we estimate for NGC $76790.2 \lesssim\left(N_{\mathrm{ph}} / N_{\text {ion }}\right)_{h v>55 \mathrm{eV}} \lesssim 20$ but the lower limit could increase if the luminosity $L\left(\left[\mathrm{O}^{+2}\right] \lambda 5007\right)$ is integrated over the whole image. The increase of the upper limit of this ratio is due to the XMM-Newton data which are $\sim 8$ times lower than ASCA/BeppoSAX ones.

Both the ratio $\left(N_{\mathrm{ph}} / N_{\mathrm{ion}}\right)_{h r>55 \mathrm{eV}}$ and the presence of weak and elusive broad $\mathrm{H} \alpha$-wings (Kewley et al. 2000) indicate a hidden AGN in the NGC 7679. To the contrary, the NGC 7679 X-ray spectrum is not highly absorbed and $N_{\mathrm{H}}<4 \times 10^{20} \mathrm{~cm}^{-2}$ (see discussion in Sect. 4.1). As a matter of fact Bian \& Gu (2006) recently found a very high detectability of hidden BLRs $(\sim 85 \%)$ for Compton-thin Sy2s with higher [O III] luminosity of $L\left(\left[\mathrm{O}^{+2}\right] \lambda 5007\right)>10^{41} \mathrm{erg} \mathrm{s}^{-1}$.

We have to note that NGC 7679 resembles, in many respects, the galaxy IRAS $12393+3520$. In this galaxy direct X-ray evidence suggests the presence of a hidden AGN (Guainazzi et al. 2000). This homology can be seen in Fig. 5 where the spectral energy distribution (SED) from the radio to the X-ray band of NGC 7679 is shown.

The composite nature of NGC 7679 is clearly seen. Whereas the starburst component dominates in the FIR-IR range, the $\mathrm{X}$-ray band emission is well below that of a typical Sy1. The extrapolation of the power-low X-ray spectrum to $13.6 \mathrm{eV}$ shows a much lower value than the typical Sy2 emission at this wavelength. This again favors the idea about a hidden central engine. Guainazzi et al. (2000) suppose that a dusty ionized absorber is able to selectively obscure the optical emission, leaving the $\mathrm{X}$-rays almost unabsorbed.

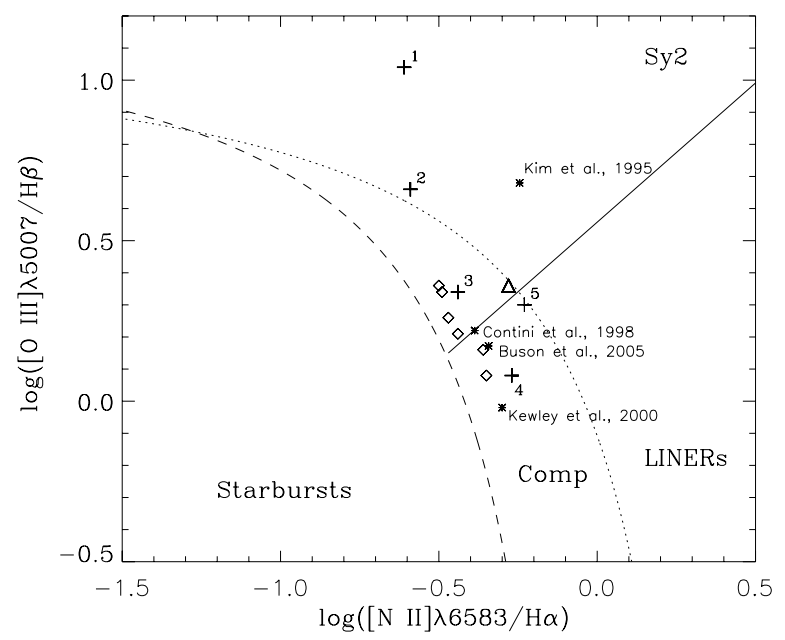

Fig. 6. The [O III $] \lambda 5007 / \mathrm{H} \beta$ vs. [N II] $] 26583 / \mathrm{H} \alpha$ diagnostic diagram of Veilleux \& Osterbrock (1987). The dashed and dotted theoretical lines demarcate between Starbursts and AGNs according to Kauffmann et al. (2003) and Kewley et al. (2001), respectively. The line dividing between LINERs and SyGs is taken according to PA02. The label "Comp" indicates the region of the diagram in which composite objects are expected to be found. The diagnostic value we measured is denoted by a thick triangle. See text for other designations.

\subsection{Ionization structure in the circumnuclear region of the NGC 7679}

The ionization map (the right panel of Fig. 4) displays the clear signature of highly-excited gas. The [O III] $\lambda 5007 / \mathrm{H} \alpha$-ratio increases in the direction of the counterpart galaxy NGC 7682 reaching a maximum of $\approx 2.5$ at about 12 arcsec off the nucleus. More than 15 years ago Durret \& Warin (1990) also reported about the presence of high-ionization gas in this direction (see their Fig. 3a), but their result seemingly did not attract attention.

On the other hand, at PA $\approx 0^{\circ}$ our map shows values around $[\mathrm{O}$ III $] \lambda 5007 / \mathrm{H} \alpha \approx 0.3$ and the ionization in this direction is entirely due to the young hot stars.

The [O III $] \lambda 5007 / \mathrm{H} \beta$ vs. [N II] $\lambda 6583 / \mathrm{H} \alpha$ diagnostic diagram (Veilleux \& Osterbrock 1987) helps to delineate the different ionization mechanisms maintaining the ionization of gaseous components in AGNs and in Starbursts. In Fig. 6 such a diagram is shown for NGC 7679. Kewley et al. (2001) distinguish between Starbursts and AGN using a theoretical upper limit derived from star-forming models. This limit is shown as a dotted line in Fig. 6. Objects with emission-line ratios above this limit cannot be explained by any possible combination of parameters in a star-forming model. Kauffmann et al. (2003) published an updated estimate for the starburst boundary derived from the SDSS observations. In Fig. 6 this boundary is shown as a dashed line. The location of the Composites is expected to lie between these two lines (see e.g. Panessa et al. 2005).

In Fig. 6 we plot the emission-line flux ratios of NGC 7679 measured in an aperture of 3 arcsec in steps of 3 arcsec both along the $\mathrm{PA} \approx 80^{\circ}$ (with crosses) and $\mathrm{PA}=0^{\circ}$ (with diamonds). The labels $1-5$ for $\mathrm{PA} \approx 80^{\circ}$ correspond to the labels in the right panel of Fig. 4. Using spectra taken from the Smithsonian Astrophysical Observatory data Center Z-Machine Archive obtained with 3 arcsec slit width, we estimate the observed $F(\mathrm{H} \alpha) / F(\mathrm{H} \beta) \sim 5$ in NLR. In Fig. 6 positions 1 and 2 at $\mathrm{PA}=80^{\circ}$ off the nucleus lie well within the region occupied by the Sy 2 galaxies. Position 5, which is at the same distance 
Table 3. The photon deficiency for unabsorbed Sy2s discussed by Panessa and Bassani (2002) and Panessa et al. (2005).

\begin{tabular}{lll}
\hline \hline galaxy & $\left(N_{\mathrm{ph}} / N_{\text {ion }}\right)_{h v>55 \mathrm{eV}}$ & $\begin{array}{l}N_{\text {rec }} / N_{\text {ion }} \\
\text { (lower limit })\end{array}$ \\
\hline ESO 540-G001 & 4.2 & 13.0 \\
CGCG 551-008 & 1.0 & \\
MCG 03-05-007 & 2.2 & \\
UGC 03134 & 19.5 & 1.2 \\
IRAS 20051-1117 & 1.6 & 2.0 \\
CGCG 303-017 & 1.3 & \\
IC 1631 & 0.3 & \\
NGC 2992 & 2.0 & \\
NGC 3147 & 0.4 & \\
NGC 4565 & 6.7 & \\
NGC 4579 & 0.2 & \\
NGC 4594 & 1.7 & \\
NGC 4698 & 0.3 & \\
NGC 5033 & 1.3 & \\
Mrk 273x & 0.4 & \\
NGC 5995 & 0.4 & \\
NGC 6221 & 0.02 & \\
NGC 6251 & 6.0 & \\
NGC 7590 & 0.4 & \\
NGC 7679 & $3.4(2.0$ from our data) & \\
\hline
\end{tabular}

from the nucleus but in opposite direction, is located nearly on the dividing line.

All points which refer to the $\mathrm{PA}=0^{\circ}$ are situated between Kauffmann's and Kewley's demarcation lines in the region of Composites.

In Fig. 6 we also plot with asterisks the nuclear diagnostic ratios according to the data of authors presented in Table 2 . The thick triangle refers to the nucleus according to our measurements under the assumption of $F(\mathrm{H} \alpha) / F(\mathrm{H} \beta)=8.5$ (Contini et al. 1998). The large scattering of nuclear values is probably due to the variations of the strength of $\mathrm{H} \beta$ absorption line of the star-forming stellar population.

\subsection{Unabsorbed SyGs with and without hidden BLRs}

The unabsorbed Sy2 galaxies with low absorption in X-rays $\left(N_{\mathrm{H}}<10^{22} \mathrm{~cm}^{-2}\right)$ possess a hidden or nonhidden central engine and BLRs. We used the [O III] $\lambda 5007$ emission to test the presence of hidden or nonhidden AGN sources in unabsorbed Sy2 galaxies in the sample of PB02 (14 objects) and Panessa et al. (2005) (6 objects selected by Moran et al. 1996) in the same way it was done for NGC 7679 (Sects. 4.1 and 4.2). We derive the ratio $\left(N_{\mathrm{ph}} / N_{\text {ion }}\right)_{h v>55 \mathrm{eV}}$ following Eqs. (1) and (2) under the assumtions of $n_{\mathrm{e}} \approx 5 \times 10^{4} \mathrm{~cm}^{-3}$ (which is an order of magnitude smaller than the critical electron density for the [O III] $\lambda 5007$ emission), $T_{\mathrm{e}} \approx 10^{4} \mathrm{~K}$, and $C F \approx 10^{-2}$. These assumptions refer to the inner circumnuclear clouds of AGNs.

The ratios are presented in Table 3. For the objects discussed in Panessa et al. (2005) the most popular (i.e. as in NED) galaxy names are used. The $L^{\text {corr }}\left(\left[\mathrm{O}^{+2}\right] \lambda 5007\right)$ values are taken from PB02 and Panessa et al. (2005). In the case of NGC 7679 we have used both their and our determinations of $L^{\text {corr }}\left(\left[\mathrm{O}^{+2}\right] \lambda 5007\right)$.

For three objects with estimated broad $\mathrm{H} \alpha$ component $L_{\mathrm{H} \alpha}^{\text {broad }}$ (Panessa et al. 2005, Table 1 therein) we also derive the number of recombinations $N_{\text {rec }}$ resulting in the $\mathrm{H} \alpha$ emission. We assume $T_{\mathrm{e}}=10^{4} \mathrm{~K}$ and $C F=1$ which leads to the estimation of the lower limit of the value of $N_{\text {rec }}$. The $N_{\text {rec }} / N_{\text {ion }}$ lower limits are also presented in Table 3.

One can see that 17 out of 20 objects of the unabsorbed Sy2s discussed here reveal $\left.N_{\mathrm{ph}} / N_{\mathrm{ion}}\right)_{h v>55 \mathrm{eV}}>0.3$. This indicates that the central AGN sources in a considerable part of the unabsorbed Sy2s are obscured. The NGC 7679 does not make an exception and also possesses a hidden AGN engine suggested both by the [O III] 55007 morphology and by the photon deficiency.

It is still not clear what kind of physical process is related to the presence of hidden central engines in Sy2s. PB02 suggest two scenarios for the unabsorbed Sy2s (i) the central engine and their BLR must be hidden by an absorbing medium with high value of the $A_{\mathrm{V}} / N_{\mathrm{H}}$ ratio, and (ii) the BLR is very weak or absent.

\section{Conclusions}

We present a new [O III] $\lambda 5007$ emission-line image of the circumnuclear region of NGC 7679 , which shows elliptical isophotes extended along the $\mathrm{PA} \approx 80^{\circ} \pm 10^{\circ}$ in the direction of the counterpart galaxy NGC 7682. The maximum of this emission is displaced by about 4 arcsec from the photometric center defined by the continuum emission.

The ratio of the quantity of ionizing photons inferred from the observed extinction corrected [O III] $\lambda 5007$ luminosity to the number of ionizing photons with $h v>55 \mathrm{eV}$ provided by the central AGN source $\left(N_{\mathrm{ph}} / N_{\mathrm{ion}}\right)_{h v>55 \mathrm{eV}} \approx 0.2-20$ as well as the presence of weak and elusive $\mathrm{H} \alpha$ broad wings probably indicate a hidden AGN.

The high ionization inferred by the flux ratio [O III $] \lambda 5007 / \mathrm{H} \alpha$ in the direction of about $\mathrm{PA} \approx 80^{\circ} \pm 10^{\circ}, \mathrm{co}-$ incides with the direction of the counterpart galaxy NGC 7682 . It is possible that the dust and gas in this direction have a direct view to the central AGN engine. It suggests that starburst and dust decay in this direction have occurred because of tidal interaction between the two galaxies.

In the direction $\mathrm{PA} \approx 0^{\circ}$, the ionization is caused entirely by hot stars.

A large part of the unabsorbed Compton-thin Sy2s with higher [O III] luminosity $\left(\gtrsim 10^{41} \mathrm{erg} \mathrm{s}^{-1}\right)$ possesses a hidden AGN source.

Acknowledgements. We are grateful to the referee, Lucio Buson, for his valuable comments which improved both the content and the clarity of this manuscript. We would like to thank T. Bonev, Institute of Astronomy of Bulgarian Academy of Sciences, for kindly providing the Fabry-Perot observations and for useful discussions. We are grateful to S. Zhekov, Space Research Institute of Bulgarian Academy of Sciences, for the numerous fruitful discussions and especially for the analysis of the X-ray properties of NGC 7679.

Our work was partially based on data from the La Palma ING, ESO NTT, and XMM-Newton Archives.

This research has made use of the SIMBAD database, operated at CDS, Strasbourg, France, and of the NASA/IPAC Extragalactic Database (NED) which is operated by the Jet Propulsion Laboratory, California Institute of Technology, under contract with the National Aeronautics and Space Administration.

We acknowledge the support of the National Science Research Fund by the grant No. F-201/2006.

\section{References}

Aldrovandi, S. M. V., \& Pequignot, D. 1973, A\&A, 25, 137

Bassani, I., Dadina, M., Maiolino, R., et al. 1999, ApJS, 121, 473

Baskin, A., \& Laor, A. 2005, MNRAS, 358, 1043

Bian, W., \& Gu, Q. 2006, ApJ, accepted [arXiv: astro-ph/0611199]

Boyle, B. J., McMahon, R. G., Wilkes, B. J., \& Elvis, M. 1995, MNRAS, 276, 315

Buson, L. M., Cappellari, M., Corsini, E. M., et al. 2006, A\&A, 447, 441

Condon, J., Huang, Z., Yin, Q., \& Thuan, T. 1991, ApJ, 378, 65 
Contini, T., Considere, S. \& Davoust, E. 1998, A\&AS, 130, 285 Della Ceca, R., Pellegrini, S., Bassani, L., et al. 2001, A\&A, 375, 781 (DC01) Draine, B. T., \& Lee, H. M. 1984, ApJ, 285, 89

Durret, F., \& Warin, F. 1990, A\&A, 238, 15

Elvis, M., Wilkes, B. J., McDowell, J. C., et al. 1994, ApJS, 95, 1

Golev, V., Yankulova, I., Bonev, T., \& Jockers, K. 1995, MNRAS, 273, 129

Golev, V., Yankulova, I., \& Bonev, T. 1996, MNRAS, 280, 29

Granato, G. L., \& Danese, L. 1994, MNRAS, 268, 235

Griffiths, R. E., Della Ceca, R., Georgantopoulos, I., et al. 1996, MNRAS, 281,

$$
71
$$

Gu, Q., Melnick, J., Fernandes, R., et al. 2006, MNRAS, 366, 480

Gu, Q. S., Huang, J. H., de Diego, J. A., et al. 2001, A\&A, 374, 932

Guainazzi, M., Dennefeld, M., Piro, L., et al. 2000, A\&A, 355, 113

Heckman, T. M., Armus, L., \& Miley, G. K. 1990, ApJS, 74, 833

Jockers, K. 1997, Exp. Astron., 7, 305

Jockers, K., Credner, T., Bonev, T., et al. 2000, Kinematika i Fizika Nebesnykh Tel, Suppl, 3, 13

Kauffmann, G., Heckman, T. M., Tremonti, C., et al. 2003, MNRAS, 346, 1055

Kewley, L. J., Heisler, C. A., Dopita, M. A., et al. 2000, ApJ, 530, 704

Kewley, L. J., Heisler, C. A., Dopita, M. A., \& Lumsden, S. 2001, ApJS, 132, 37

Kim, D.-C., Sanders, D. B., Veilleux, S., Mazzarella, J. M., \& Soifer, B. T. 1995, ApJS, 98, 129

Kotilainen, J. K., \& Prieto, M. A. 1995, A\&A, 295, 646

Levenson, N., Weaver, K., \& Heckman, T. 2001, ApJ, 550, 230

Lipari, S., Bonatto, Ch., \& Pastoriza, M. 1991, MNRAS, 253, 19

Mas-Hesse, J. M., Rodriguez-Pascual, P. M., Sanz Fernandez de Cordoba, L., et al. 1995, A\&A, 298, 22
Moran, E. C., Halpern, J. P., \& Helfand, D. J. 1996, ApJS, 106, 341

Moustakas, J., \& Kennicutt, R. C. 2006, ApJS, 164, 81

Osterbrock, D. 1989, Astrophysics of gaseous nebulae and active galactic nuclei (University Science Books)

Panessa, F., \& Bassani, L. 2002, A\&A, 394, 435 (PB02)

Panessa, F., Wolter, A., Pellegrini, S., et al. 2005, ApJ, 631, 707

Pier, E. A., \& Krolik, J. 1992, ApJ, 401, 99

Pogge, R. W. 1989, AJ, 98, 124

Risaliti, G. 2002, A\&A, 386, 379

Risaliti, G., Maiolino, R., \& Salvati, M. 1999, ApJ, 522, 157

Rosati, P., \& Chandra Deep Field South Team 2001, 199th AAS Meeting, BAAS, 33,1519

Sanders, D., Soifer, B., Elias, J., et al. 1988, ApJ, 325, 74

Schmitt, H. R., Kinney, A. L., Calzetti, D., \& Storchi Bergmann, T. 1997, AJ, 114,592

Simpson, C., Mulchaey, J. S., Wislon, A. S., Ward, M. J., \& Alonso-Herrero, A. 1996, ApJ, 457, L19

Simpson, C., Wislon, A. S., Bower, G., et al. 1997, ApJ, 474, 121

Smith, H. E., Lonsdale, C. J., \& Londsdale C. J. 1998, ApJ, 492, 137

Stine, P. C. 1992, ApJS, 81, 49

Telesco, C. M., Dressel, L., \& Wolstencroft, R. 1993, ApJ, 414, 120

Veilleux, S., \& Osterbrock, D. E. 1987, ApJS, 63, 295

Veilleux, S., Kim, D.-C., Sanders, D. B., Mazzarella, J. M., \& Soifer, B. T. 1995, ApJS, 98, 171

Wilson, A. S., Braatz, J. A., Heckman, T. M., Krolik, J. H., \& Miley, G. K. 1993, ApJ, 419, L61

Yankulova, I. 1999, A\&A, 344, 36 